\title{
Advancements in organic nonvolatile memory devices
}

\author{
LIU Xin ${ }^{1,2}$, JI ZhuoYu $^{1}$, LIU Ming ${ }^{1 *}$, SHANG LiWei ${ }^{1}$, LI DongMei ${ }^{1} \&$ DAI YueHua ${ }^{2}$ \\ ${ }^{1}$ Laboratory of Nanofabrication and Novel Device Integration, Institute of Microelectronics, Chinese Academy of Sciences, Beijing 100029, \\ China; \\ ${ }^{2}$ School of Electronic and Information Engineering, Anhui University, Hefei 230039, China
}

Received April 20, 2011; accepted July 25, 2011

\begin{abstract}
As one of the most promising candidates for next generation storage media, organic memory devices have aroused worldwide research interest in both academia and industry. In recent years, organic memories have experienced rapid progress. We review the development of organic resistive switching memories in terms of structure, characteristics, materials used, and integration. Some basic concepts are discussed, as well as the obstacles hindering the development and possible commercialization of organic memory devices.
\end{abstract}

organic memory, two-terminal memory devices, organic thin-film transistors, integration, multilevel effect

Citation: $\quad$ Liu X, Ji Z Y, Liu M, et al. Advancements in organic nonvolatile memory devices. Chinese Sci Bull, 2011, 56: 3178-3190, doi: 10.1007/s11434-011-4695-5

Because of their potential application in low-cost, flexible, large area, and lightweight organic electronics, organic materials have attracted considerable attention in many areas such as organic thin-film transistors (OTFTs) [1-9], organic light emitting diodes [10-12], and organic photovoltaic cells [13-15], and significant progress has been made during the last few years. As a key component of organic integrated circuits, organic nonvolatile memory has experienced rapid development recently.

The field of organic memories is considered to be relatively new, with several high profile articles popularizing the field having appeared only within the past decade. However, the origins of related work date back to the early 1970s when thin films of organic materials were shown to exhibit electrical switching capabilities. Organic materials are promising candidates for future information technologies, since many organic electronic devices exhibit switching characteristics, and have therefore been proposed as the basis for organic nonvolatile memory technology. Most research work has focused on the storage medium itself, ranging from amorphous to crystalline phase change materials, ferroelectrics, and electrochemically reduced or oxi-

*Corresponding author (email: liuming@ime.ac.cn) dized materials. However, in terms of application, organic memory devices are often characterized by their performance, and materials with excellent reliability need to be developed. Great progress has been made in the integration of organic memory devices during the past few years. In 2003, Hewlett-Packard Labs, in collaboration with UCLA, fabricated a crossbar memory circuit of 64 bits $(8 \times 8)$ at a density of $5.9 \mathrm{Gbit} / \mathrm{cm}^{2}$ using nanoimprint lithography [16,17]. In 2004, Hewlett-Packard Labs fabricated a 1 Kbit $(34 \times 34)$ crossbar circuit (with line width $35 \mathrm{~nm}$ and halfpitch $50 \mathrm{~nm}$ ) at a density of $10 \mathrm{Gbit} / \mathrm{cm}^{2}$ [18], as shown in Figure 1. Then in 2005, Hewlett-Packard Labs reported the fabrication of a $1 \mathrm{Kbit}$ crossbar molecular memory at a density of $28 \mathrm{Gbit} / \mathrm{cm}^{2}$ (30-nm half-pitch) [19]. In 2007, the Health research group of Caltech refreshed certain world records. They demonstrated a 160 Kbit $(400 \times 400)$ crossbar memory circuit using the superlattice nanowire pattern transfer (SNAP) method, with a density of $10^{11} \mathrm{~b} / \mathrm{cm}^{2}$ (line width $16 \mathrm{~nm}$ and $33 \mathrm{~nm}$ pitch) [20]. Recently, nonvolatile memory arrays that can endure more than 1000 program and erase cycles with 676 organic floating-gate transistors arranged in a $26 \times 26$ array on a $125 \mathrm{~mm}$ thick plastic film, have been realized by Sekitani et al. [21].

Organic nonvolatile memory devices generally fall into 

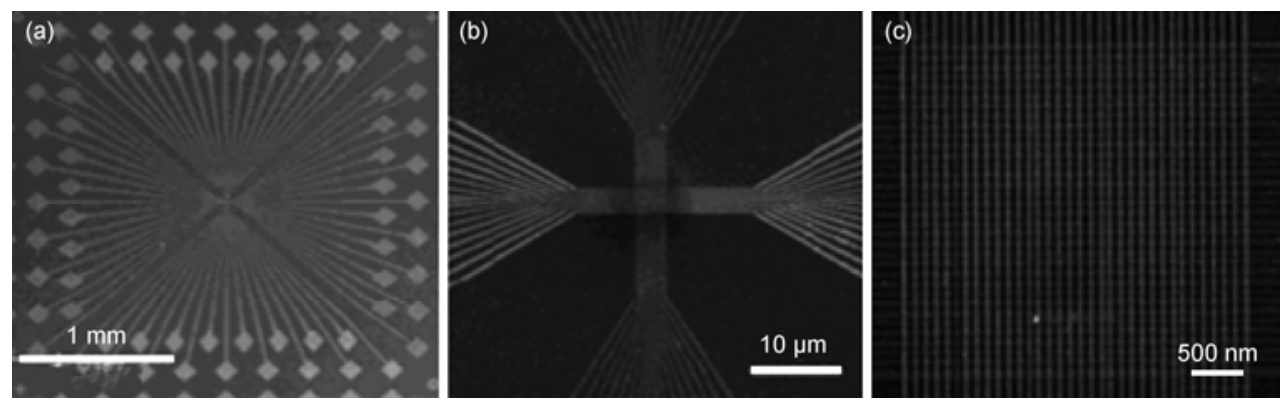

Figure 1 (a) SEM images of an array fabricated on the glass substrate; (b) two perpendicular arrays of nanowires connected to micron-scale wires; (c) $34 \times 34$ nanowire crossbar structure [18]

two categories; the two-terminal structure, which usually refers to resistive memory devices, and the thin-film transistor (TFT) structure. In this feature article, we first introduce two-terminal memory devices together with their integration and multilevel effect, which is a recent hot issue in this field. Then, we focus on organic thin-film transistor memory devices and discuss their application. Finally, the obstacles hindering the development and possible commercialization of organic memory devices are discussed.

\section{Two-terminal memory devices}

Two-terminal memory devices have the advantage of a simple process and high-density storage through the crossbar structure. Most of the reported organic memory devices adopted a two-terminal structure with the active medium (triple layer or single layer) sandwiched between two electrodes. To obtain high-density storage, the two-terminal memory devices are mostly fabricated with cross-point array structure. However, the cross-talk phenomenon appears simultaneously. Investigation of the organic memory integrated circuit has provided a solution for this.

\subsection{Two-terminal organic memory devices}

In recent years, much attention has been paid to two-terminal memory devices based on organic molecular and polymer materials [22-24] owing to the high potential of using these devices in nonvolatile memory. A characteristic of these memory devices is electrical bistability, which means switching between the high-resistance state (OFF state) and low-resistance state (ON state) under some applied condition, reading out by means of a lower voltage to probe the resistance state of the device, and having either state remain for a prolonged time (retention time) even after removing the external electrical field. The memory effect has been demonstrated in various novel device architectures and material systems, such as triple layer (organic/metal nanoparticle/organic (OMO)) [25,26] or single layer sandwiched between two electrodes. Single layer active material systems include polymer-metal nanoparticle blend systems [27,28], all organic donor-acceptor systems [23], and single-component materials [29-31]. The materials used, and electrical behavior and mechanisms of these devices are discussed in detail in the following paragraphs.

Both small conjugated molecules and polymers have been employed as the organic semiconductor materials in these devices. The chemical structure of some of the materials presented in this section is listed in Figure 2. Material selection is critical as only an appropriate combination of materials will create the desired memory effect; for example, combining the organic donor material with acceptor material, introducing charge trapping dopants (e.g., metal, metal oxides, etc.) into an organic medium, or charge transfer between the composite materials, causes the device to exhibit memory function. Materials that are used in the triplelayer structure include AIDCN [25], tris-(8-hydroxyquinoline) aluminum $\left(\mathrm{Alq}_{3}\right)$ [32], cyanopolyfluorene [33], and pentacene [26]. Single-layer devices using either small organic compounds or polymers have also been demonstrated. Small organic compounds include pentacene [26], codeposited Cu:TCNQ (TCNQ:7,7,8,8-tetracyanoquinoline) [34], $\mathrm{Alq}_{3}[29,35], \mathrm{N}, \mathrm{N}^{\prime}$-diphenyl-N,N'-bis(1-naphthyl)-(1, 1'-biphenyl)-4,4'-diamine (NPB) [36], oxotitanium phthalocyanine (TiOPc) [37], and the polymers of polyaniline (PANI) [30], 8-hydroxyquinoline (8HQ) [27], poly [3-(6methoxyhexyl)thiophene] [31], tetrathiafulvalene (TTF), and [6,6]-phenyl $\mathrm{C}_{61}$-butyric acid methyl ester (PCBM) [23], poly(3,4-ethylene-dioxythiophene): polystyrenesulfonate (PEDOT:PSS) [38]. Based on the different materials, it is possible to divide existing organic memory devices into three categories: charge-transfer complex memory devices, metallic nanoparticle (NP) doped memory devices, and all organic system memory devices.

(1) Charge-transfer complex memory devices. In 1979, Potember and Poehler [39] first observed the switching phenomenon in a Cu:TCNQ charge-transfer (CT) complex with a sandwiched structure of $\mathrm{Cu} / \mathrm{Cu}: \mathrm{TCNQ} / \mathrm{Al}$, where the field induced solid-state reversible phase transition is postulated for this effect. They fabricated Cu:TCNQ CT-complex films with a solution growth method on a precleaned $\mathrm{Cu}$ substrate dipped into a solution of degassed acetonitrile saturated with TCNQ. However, most devices fabricated by 
<smiles>N#Cc1nc(N)[nH]c1C#N</smiles>

(a)

(d)

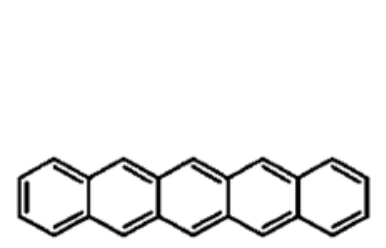

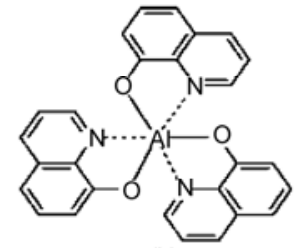

(b)

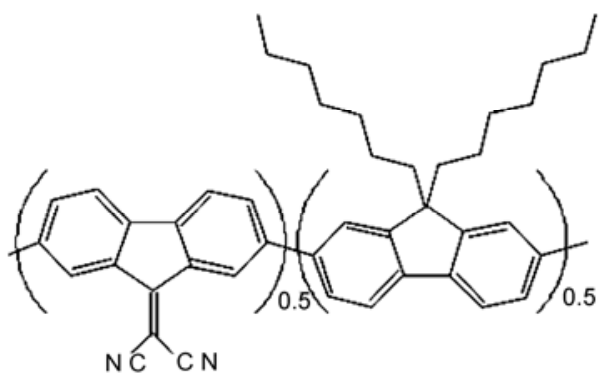

(c)

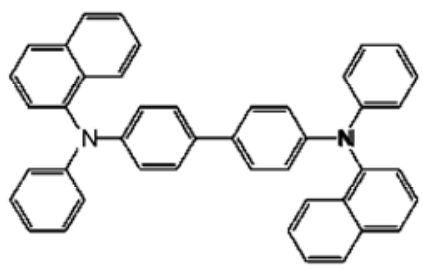

(e)<smiles>Oc1cccc2cccnc12</smiles>

(f)<smiles>C1=CSC(=C2SC=CS2)S1</smiles>

(h)

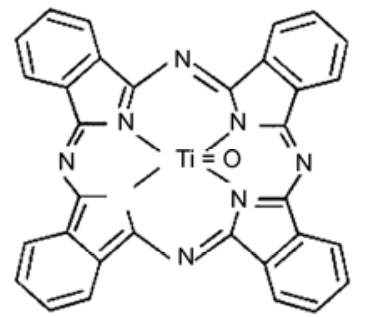

(k)

Figure 2 Chemical structure of (a) 2-amino-4,5-imidazoledicarbonitrile (AIDCN), (b) tris-(8-hydroxyquinoline) aluminum (Alq $)_{3}$, (c) cyanopolyfluorene, (d) pentacene, (e) N,N'-diphenyl-N,N'-bis(1-naphthyl)-(1,1'-biphenyl)-4,4'-diamine (NPB), (f) 8-hydroxyquinoline (8HQ), (g) polyaniline (PANI), (h) tetrathiafulvalene (TTF), (i) [6,6]-phenyl $\mathrm{C}_{61}$-butyric acid methyl ester (PCBM), (j) poly(3,4-ethylene-dioxythiophene): polystyrenesulfonate (PEDOT:PSS), and (k) oxotitanium phthalocyanine (TiOPc).

this solution method have a serious problem with homogeneity in the Cu:TCNQ films, leading to a lack of reproducibility in device characteristics.

Motivated by the conductive characteristics of organic charge-transfer complexes, a wide range of charge-transfer complexes have been studied, such as AgTCNQ and $\mathrm{C}_{60} \mathrm{TCNQ}$ with TCNQ as the electron acceptor $[40,41]$ and other organic acceptors, e.g., AIDCN and $\mathrm{ZnPc}$ coupled with $\mathrm{Cu}$ [42]. There are many new ways to prepare organic thin films, including the Langmuir-Blodgett method and sublimation methods, such as organic molecular beam epitaxy and vacuum evaporation. In 2003, Oyamada et al. [34] fabricated a reproducible switching device with an ITO/ $\mathrm{Al} / \mathrm{Al}_{2} \mathrm{O}_{3} / \mathrm{Cu}: \mathrm{TCNQ} / \mathrm{Al}$ configuration. Uniform $\mathrm{Cu}$ :TCNQ CT-complex films were deposited with various ratios of Cu:TCNQ (0:1, 1:2, 1:1, 3:2, 2:1, 4:1 atm/mol\%) via thermal codeposition. In particular, the Cu:TCNQ (1:1 atm/ mol\%) thin film resulted in the largest ON/OFF ratio of $\sim 10^{4}$. It has been reported that the interface of the $\mathrm{Al}$ anode and $\mathrm{Cu}: \mathrm{TCNQ}$ layers is mainly responsible for the electrical switching. In 2008, our group [41] proposed a bistable device with a uniform AgTCNQ thin-film layer sandwiched in a Ti/AgTCNQ/Au crossbar structure. We obtained a uniform AgTCNQ film by alternating vacuum evaporation of an $1 \mathrm{~nm} \mathrm{Ag}$ layer and a $14.6 \mathrm{~nm}$ TCNQ layer ten times. The device exhibited a reversible and reproducible memory effect with a lower operation voltage $(<5 \mathrm{~V})$ than that with a CuTCNQ active layer.

(2) Metallic nanoparticle doped memory devices. Metallic nanoparticle doped devices realize memory function by embedding metal thin-film or doping metal NPs within the organic layer(s). The embedded metal thin-film or doped metal NPs generally act as a charge trapping center, modulating the resistance state of the devices under an appropriate bias condition.

The metal thin films are usually embedded into an or- 
ganic layer by evaporation, so these devices form multi-layer stacking structures. Al has been widely used as the middle metal layer and has shown stable memory characteristics with various organic materials. Yang's group $[25,43]$ first proposed an organic bistable device with the OMO structure, using AIDCN and $\mathrm{Al}$ as the embedded organic layers and metal layer, respectively (see Figure 3). The writing and erasing process happened at biases of different polarities, i.e., bipolar switching (as shown in Figure 4). It has been demonstrated that the switching behavior only occurs in the presence of the embedded thin metal layer and if the thickness of the embedded layer is less than 10 $\mathrm{nm}$. The switching mechanism is interpreted as the trapping and retrapping of carriers occurring in the inner metal layer, while the mechanism of conducting filament formation is denied.

In 2004, Bozano et al. [32] demonstrated a similar bistable effect in organic devices exhibiting unipolar resistance switching behavior, with a thin metal layer located in the middle of the Alq3 layer. Beginning at the ON state, a negative differential resistance (NDR) region appeared when voltage was ramped up from zero (as shown in Figure 5), promising a potential application in multilevel memories. To prove the dependence of the switching behavior on the inner metal layer, they prepared other devices with different thicknesses of inner metal layers $(5-40 \mathrm{~nm})$ and using different metals ( $\mathrm{Al}, \mathrm{Cr}, \mathrm{Cu}, \mathrm{Mg}, \mathrm{Ag}$ ). Only devices with metal layers less than $10 \mathrm{~nm}$ exhibited switching behavior. Recently, Reddy et al. [44] investigated the effect of the thickness of the intermediate metal layer and the size of the

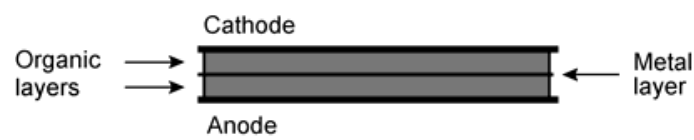

Figure 3 Structure of an electrical device [25].

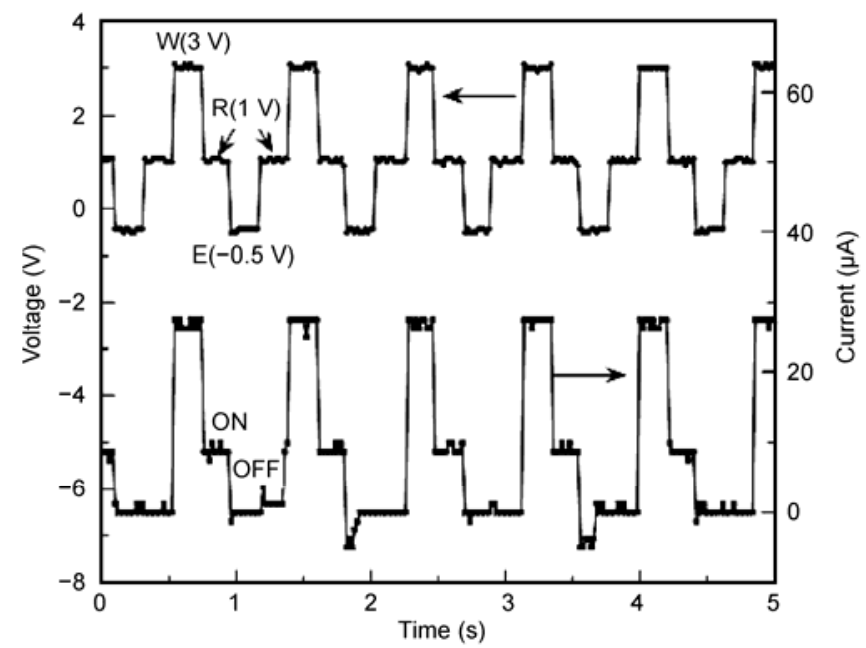

Figure 4 Typical current responses of an OBD during the write-readerase voltage cycle [25]. nanoparticles on device performance. They fabricated a trilayer $\mathrm{ITO} / \mathrm{Alq}_{3} / \mathrm{Al}$ nanoislands/Alq $/ \mathrm{Al}$ memory device, with the thickness of each $\mathrm{Alq}_{3}$ layer about $50 \mathrm{~nm}$ and that of the middle Al layer varying between 5 and $20 \mathrm{~nm}$. To obtain devices with well controlled bistability, the middle aluminum layer should have an island-like structure, instead of being a continuous metal layer. In general terms, the morphology and the thickness of the middle metal layer depend on the evaporation rate. A deposition rate less than $0.1 \mathrm{~nm} / \mathrm{s}$ was adopted to obtain island-like growth and bistable devices. Devices with a $10 \mathrm{~nm}$ thick middle Al layer showed the largest ON/OFF current ratio $\left(>10^{5}\right)$. When the $\mathrm{Al}$ layer is increased beyond $10 \mathrm{~nm}$, the ON/OFF ratio decreases sharply and the switching properties almost vanish at a thickness of $20 \mathrm{~nm}$. Simmons et al. [45] interpreted the NDR of their device in terms of the Simmons and Verderber (SV) model. Charge trapping on the metal NPs and the resulting space-charge field inhibition of charge injection was attributed to the resistive switching. This charge-trapping mechanism was also adopted by various other groups $[37,46]$. Tang et al. [47], however, argued that the SV model may not be a good one for conductance switching as it disregards potential energy changes resulting from the charging process. They believed that the electrical behavior in their devices was caused by 2D single-electron tunneling by nanometer-sized metal islands, formed unexpectedly by the nucleation and growth of a metal thin-film electrode.

The organic memory device has taken a giant leap forward in technology by simplifying the layered structure into a single-layer polymer memory device, which can be formed by solution processing. The nonvolatile memory function is realized using a polymer film blended with organic compounds and metal NPs. Au is effective as a nanocrystal and stable memory characteristics are realized in $\mathrm{Au}$ based organic bistable devices. In 2004, Ouyang et al. [27] fabricated a simple memory cell with a single layer polymer

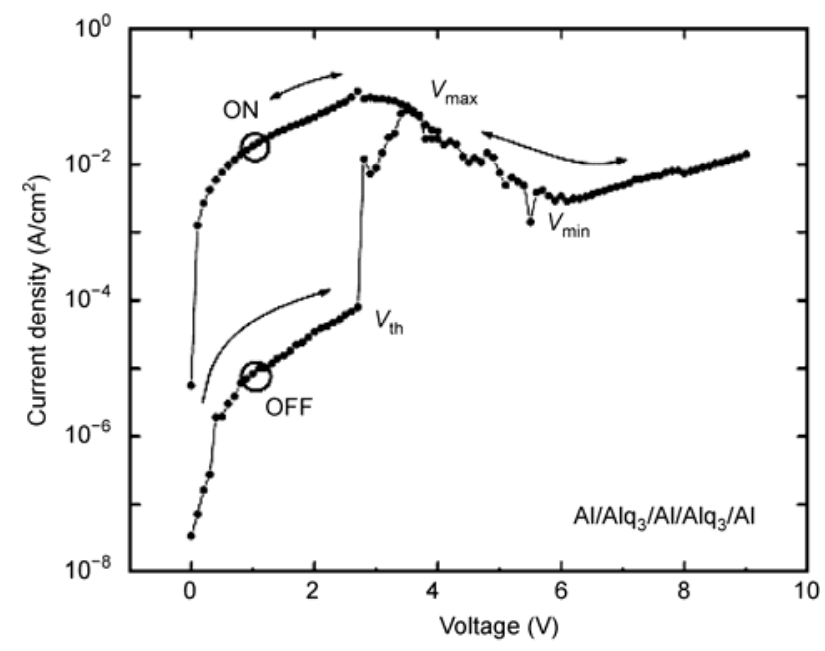

Figure 5 Current-voltage characteristic of an $\mathrm{Al}(50 \mathrm{~nm}) / \mathrm{Alq}_{3}(50 \mathrm{~nm}) /$ $\mathrm{Al}(5 \mathrm{~nm}) / \mathrm{Alq}_{3}(50 \mathrm{~nm}) / \mathrm{Al}(50 \mathrm{~nm})$ device [32]. 
film sandwiched between two Al electrodes. The polymer film was formed by spin-coating a 1,2-dichloroben-zene solution of 1-dodecanethiol-protected gold nanoparticles (Au-DT NPs), 8-hydroxyquinoline (8HQ), and polystyrene (PS). It was shown that $8 \mathrm{HQ}$ and gold NPs act as electron donors and acceptors, respectively. The stability of the negative charge on the gold NPs is due to the insulating coating on the gold NPs (DT), which prevents recombination of the charge after removal of the external electric field. PS may act merely as an inert matrix for Au-DT NPs and 8HQ and does not play a role in the electronic transition mechanism. A little while later, Ouyang et al. [48] demonstrated a write-once read-many times (WORM) memory device by simply replacing the capping of the Au NPs with 2-naphthalenethiol (2NT). The transition mechanism was postulated as being the electric-field-induced charge-transfer between the Au NPs and the capping 2NT. In 2006, Prakash et al. [49] demonstrated a polymer memory device with an active layer consisting of conjugated poly(3-hexylthiophene) (P3HT) and gold nanoparticles capped with 1-dodecanethiol, of which the conjugated polymer serves as both the matrix and active component of the device. The devices exhibited excellent endurance (>1500 cycles) and retention (>13 h). In 2007, Song et al. [50] reported a similar nonvolatile memory device using poly(N-vinylcarbazole) (PVK) containing Au NPs as the active layer, with a high ON/OFF current ratio up to $10^{5}$. In 2007 , Tseng et al. [51] reported a charge-transfer effect in the polyaniline (PAIN) nanofiber/gold nanoparticle nanocomposite memory system. The device exhibited very fast response time ( $<25 \mathrm{~ns})$, with the electric-field-induced charge-transfer between the conjugated PAIN and gold nanoparticle responsible for the electrical conductance switching.

(3) All organic system memory devices. One of the problems of metal thin-film systems or $\mathrm{Au}$ nanoparticle systems is the phase separation that occurs between the organic (or polymer) material and the metal nanoparticles. This phase separation prevents the device from having a stable and long operating time. Thus, all organic system memory devices were demonstrated, and we refer to these memory devices with single-component materials [29,35,36] or all organic donor-acceptor (D-A) systems [23] as an active layer sandwiched between two electrodes.

In fact, significant research has been carried out on this type of device since the 1970s. The focus was initially on various polymer films [52-54]. In the work by Carchano et al. [52], both electrodes were gold $(\mathrm{Au})$ and the organic insulator was glow-discharge-deposited polystyrene. The resistance ratio was reported to be more than $10^{7}$ and the cycle endurance was more than 2000 times. With a polymer thickness of less than $150 \mathrm{~nm}$, the threshold voltage increased linearly with the polymer thickness. Switching in other polymers formed by glow-discharge from acetylene, benzene, and aniline [55] has also been observed, with a wide variety of threshold behaviors and various degrees of damage observed in the electrodes after switching.

Memory effect has been demonstrated in many singlecomponent materials, such as pentacene [26], TiOPc [36], NPB [37], PEDOT:PSS [38], $\mathrm{Alq}_{3}$ [56], and PVK [57]. In 2004, Tondelier et al. [26] studied both the triple-layer and single-layer structures based on pentacene. Similar switching behavior was found even without the middle metal nanocluster layer. The switching behavior was attributed to the inclusion of metal nanoparticles into the organic material during the top electrode evaporation for both types of devices, which resulted in nano-filamentary metallic pathways forming through the polymer under high electric fields, giving rise to a high conductivity ON state. In 2005, Lai et al. [57] fabricated a bistable memory device by spin-coating the PVK films. The PVK films exhibit good retention and a high ON/OFF resistance ratio of $10^{4}$, with the filament theory used [38] to elucidate the switching mechanism. Recently, our group proposed a resistive switching device with a single-layer PEDOT:PSS film as the active material. The device displayed a nonpolar property, which means that switching between the high resistance state (OFF-state) and low resistance state (ON-state) does not depend on the polarity of the applied voltage bias. The switching mechanism was attributed to the forming and rupture of the conductive paths in the PEDOT:PSS film.

Organic D-A complexes, exhibiting electrical bistability and versatility in molecular design, have also received considerable attention; for example, a simple mixture of the acceptor and donor, biscyanovinyl-pyridine and decacyclene [58] or nitro-benzylmalonitrile and phenylenediamine [59], as well as a complex with the acceptor and donor blended into a host polymer, soluble fullerene (PCBM) and tetrathiafulvalene (TTF) in polystyrene [31]. It is interesting that a blend of two acceptors, PCBM and TCNQ, has been shown to have the switching property. In 2006, Liu et al. [60] fabricated an electrically bistable memory device based on the spin-coated PCBM/TCNQ complex thin film. The ON/OFF ratio was as high as $10^{6}$ and either state was found to remain stable for more than five months, even after the external electric field had been removed. They explained that with an appropriate molecular film thickness $(\sim 100 \mathrm{~nm})$, molecular quantum dot devices formed, and the quantum dot tunneling was assumed for the switching characteristics. Hitherto, almost all research on D-A systems has focused on the exploration of different D-A complexes with better electrical properties for data storage. Recently, Ma et al. [61] investigated the influence of the electron donor and acceptor units on the electrical function of materials by synthesizing a new series of D-A molecules with similar electron donor and acceptor units. This work is an important step towards rational design of new organic D-A molecules with suitable structures for high-performance data storage.

In addition to developing new material systems similar to the above three categories, recently research interests have focused on the effect of various electrode materials on or- 
ganic memory devices [62,63]. Ha and Kim [62] reported memory devices based on PEDOT:PSS thin film with different bottom electrodes (BEs) and top electrodes (TEs). The BEs were indium tin oxide (ITO) and Al, while the TEs were $\mathrm{Al}, \mathrm{Ti}, \mathrm{Cr}$, ITO, $\mathrm{Au}, \mathrm{Ni}, \mathrm{Pd}$, and $\mathrm{Pt}$. The ITO/ PEDOT:PSS/TE devices only had bipolar switching behavior, while the Al/PEDOT:PSS/TE devices did not have any switching behavior unless a compliance current was used in the write-operation method, in which case they had a unipolar switching behavior regardless of the TE material. Ouyang et al. [63] proposed electrode-sensitive bipolar resistive memory devices with a polymer film containing $\mathrm{Au}-2 \mathrm{NT}$ NPs sandwiched between $\mathrm{Al} \mathrm{BE}$ and $\mathrm{Au}, \mathrm{Cu}, \mathrm{Al}$ TEs, respectively. It has been concluded that the absolute value of the threshold voltage has a close relationship with the TEs' work function. The electrode sensitivity of the resistive switching has been attributed to the contact potential between Au-2NT NPs and a metal electrode arising from their different work functions.

Great progress has been made in the structure, performance, and fabrication methods for organic memory devices, but many controversies still exist with regard to the conducting mechanisms. There are classical theory models for inorganic materials, many of which have been modified to explain the phenomenon of organic materials. It is difficult but meaningful to establish mathematical or numerical models for organic semiconductor materials, which will be of great help in improving the performance of organic memory devices, and thus promoting their practical applications.

\subsection{Integration of two-terminal organic memory devices}

Over the past decade, many research groups have tried to develop novel organic materials, optimal device structures, and fabrication methods for practical organic memory applications, and many high-performance devices have been reported. However, from a practical application point of view, it is critical to realize simple high-density memory circuits that rely closely on integration technology.

To achieve high-density storage, polymer nonvolatile memory devices are scaled down and fabricated in a crossbar array structure where the memory cells are weakly isolated from each other, so crosstalk and misreading [64] in memory operations are inevitable. Figure 6 shows how a misreading may occur in the read operation of a crossbar memory array. If three neighboring points $\left(A A^{\prime}, B B^{\prime}, C C^{\prime}\right)$ in a $2 \times 2$ crossbar matrix are in the $\mathrm{ON}$ state, then the fourth point $D D^{\prime}$ will also be read as being in the ON state, whether or not it is in the OFF state, because the current can flow through the path $D^{\prime} \rightarrow B^{\prime} B \rightarrow A A^{\prime} \rightarrow C^{\prime} C \rightarrow D$ [65-68]. Thus, it is necessary to implant switch components such as transistors or diodes to prevent unexpected crosstalk. The single-transistor and memory (one transistor and one resistor; 1T1R) and single-diode and memory (one diode and one resistor; 1D1R) structures are promising building blocks to achieve random accessibility in polymer nonvolatile memory applications.

In the $1 \mathrm{~T} 1 \mathrm{R}$ structure, a resistive memory device is series connected to the drain of the transistor, allowing the current through the memory device to be controlled by the gate voltage $\left(V_{\mathrm{g}}\right)$, thereby preventing damage induced by excess current to the memory device. Kim et al. [69] reported a hybrid device with a silicon transistor and a resistive polymer memory element. These devices have a retention time of more than $10^{4}$ and an $I_{\text {on }} / I_{\text {off }}$ ratio of more than $10^{3}$. Although a large number of studies have been carried out in the inorganic field of 1T1R memory circuitry [65-67,70], because of the poor processability of organic materials, it is generally difficult to fabricate both organic transistors and organic resistors in one chip without damaging the active layers and with no degradation of the device. Thus, the feasibility of fabricating an all organic 1T1R memory circuit deserves in-depth exploration.

For the 1D1R structure, the diode actually acts as a rectifier to improve the misreading phenomenon. It has been reported that the larger the rectifying ratio, the smaller the possibility of misreading [68]. In 2009, Lin et al. [71] proposed a UV-erasable stacked nonvolatile memory device with the 1D1R structure. The functions of UV erasing, induced by carriers escaping within the memory layer, and the diode switch can greatly simplify the required peripheral circuits. The data stored in the memory can be retained for (a)

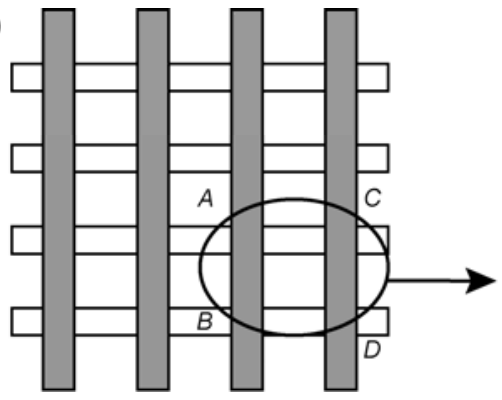

(b)

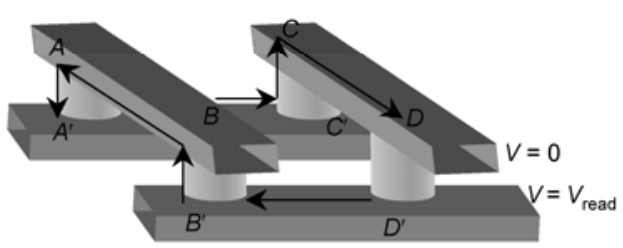

Figure 6 Misreading phenomenon in crossbar memory: (a) simple design with a matrix of switching devices, and (b) the current path leading to misreading. 
more than $10 \mathrm{~d}$ in the ambient air, while the estimated retention time is nearly one year. Thus, the authors believe this device to be fully applicable to RFID tags, in which data can be read, written, and retained for the product's entire lifetime. In 2009, Teo et al. [72] reported a organicbased 1D1R memory device as shown in Figure 7, with a high on/off current ratio of $10^{6}$ and a high rectification ration of $10^{3}$. Regarding the permanent conformational change of the material used as the memory layer when it undergoes a threshold voltage, the memory displayed only write-once-read-many times (WORM) behavior. This limits its application because rewritability is essential for most data storage applications. Recently, Cho et al. [73] solved this problem by introducing a unipolar-type memory element, because the 1D1R device with bipolar memory cannot operate properly since it is not erasable because of the suppressed current at the reverse polarity. They constructed array-type 1D-1R memory devices combining an inorganic Schottky diode and organic unipolar memory (as shown in Figure 8), which presented good retention characteristics $\left(10^{4} \mathrm{~s}\right)$ with extrapolated retention times of more than 1 year and endurance cycling of more than 280 times.

\subsection{Multilevel effect of two-terminal organic memory devices}

With the rapid development of information technology, the demand for inexpensive and high density data storage media has increased. The traditional way of achieving a low cost per bit is through the scaling size or multilevel storage, although multilevel storage has attracted more and more attention because of the fact that the scaling method is limited by photolithography [74]. The future goal of the memory cell is to obtain multibit information from a small cell by generating $2^{n}$ threshold-voltage $\left(V_{\mathrm{th}}\right)$ levels for $\mathrm{n}$ bits per cell [75]. All the studies mentioned above have focused on one-bit storage (with a 0 or 1 state). In this section, we discuss the development of a more promising way to improve storage density, namely multibit storage, and its applications.

The discovery of multilevel storage can be traced back to the 1960s, when Simmons and Verderber [45] reported multi-stability in $\mathrm{SiO}_{2}$ films with electroforming as a result of the diffusion of $\mathrm{Au}$ particles upon evaporation forming an impurity band of charge-transport levels as well as deeper charge-trapping levels. Charging of these levels under (a)

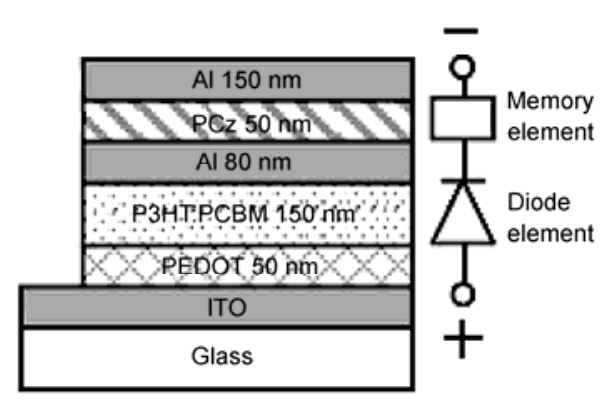

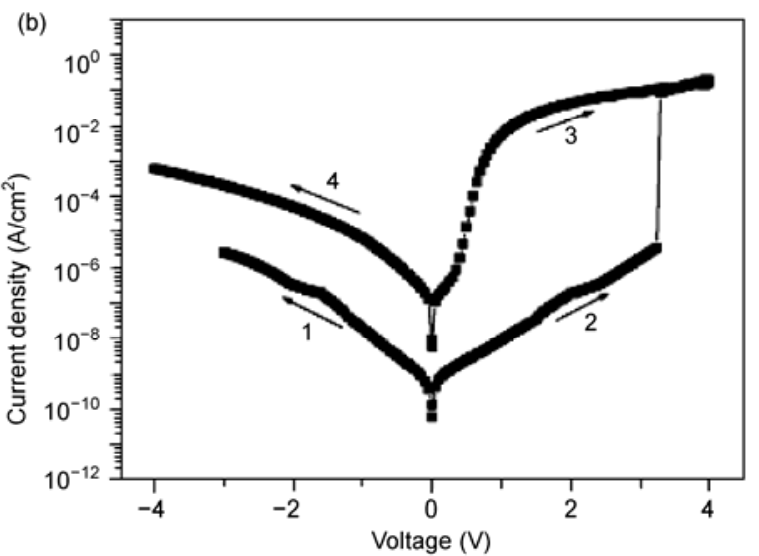

Figure 7 (a) Schematic and (b) $J-V$ characteristic of the rectifying diode-memory device [72].

(a)

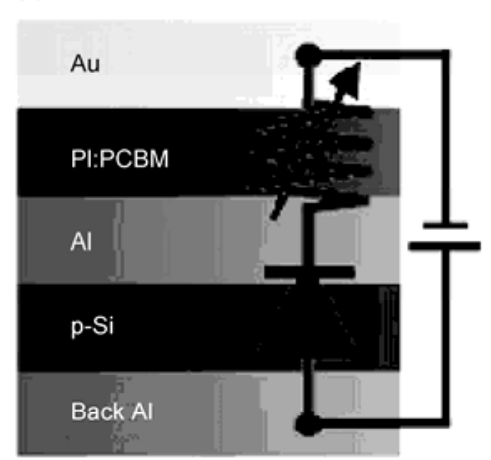

(b)

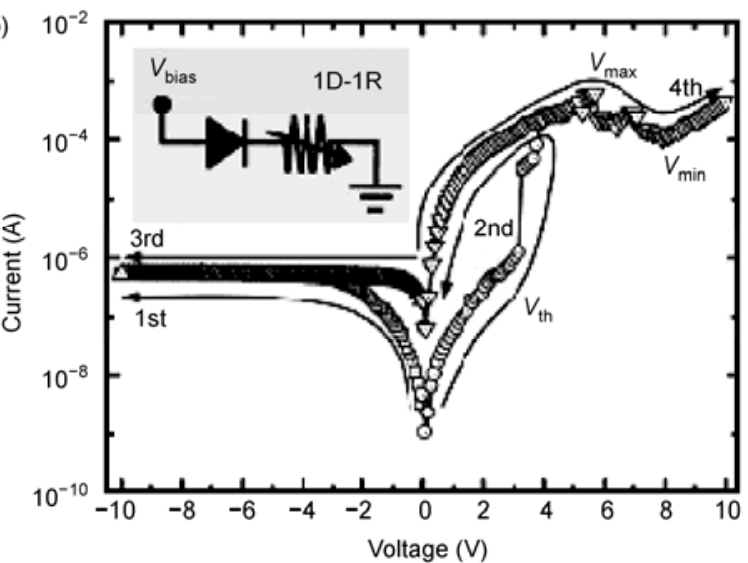

Figure 8 (a) Schematic of the layered structure, and (b) $I-V$ characteristics of the 1D1R structure [73]. 
bias leads to an $\mathrm{N}$-shaped current curve, which is seen as the negative differential resistance (NDR) region.

In recent years, many researchers have demonstrated multilevel conductance switching correlating closely with the NDR effect based on different organic materials [76-80]. In 2005, Majumdar et al. [76] demonstrated a bistable device and WORM device merely by changing the concentration of fullerenes $\left(\mathrm{C}_{60}\right)$ in a polystyrene insulating matrix (PS: $\mathrm{C}_{60}$ ), with both devices exhibiting the NDR effect. If a certain bias between the NDR regimes were applied, the consequent read voltage probes would show a device history, leading to a multi-stability response of the device as shown in Figure 9. They explained the device physics based on a mechanism similar to tunneling and impurity band conduction. Recently, Park et al. [78] carried out an outstanding work. They proposed a vertically double-stacked $4 \mathrm{~F}^{2}$ nonvolatile memory cell that can operate eight current levels, corresponding to double-stacked multilevel cell (four-level cell) or three-bit memory cell behavior (Figure 10). All the fabrication processes for their device were carried out in a multichamber evaporator without breaking the vacuum, thereby avoiding contamination (e.g., $\mathrm{H}, \mathrm{O}, \mathrm{N}, \mathrm{Cl}$, and $\mathrm{F}$ atoms). This device showed excellent performance, thus demonstrating the feasibility of terabit-level nonvolatile small-molecule memory. In light of the above investigation, it seems that multilevel storage could be achieved if the materials show NDR behavior. However, not all organic semiconductor materials have the NDR effect. Hence, efforts have been made to look for suitable molecules or systems exhibiting this phenomenon as well as the mechanism for NDR [81-83].

There are also different explanations for the formation of the multilevel effect. Bandyopadhyay et al. [84] found multilevel conductance switching in supramolecular structures of Rose Bengal molecules, the mechanism of which was attributed to a combination of electrochemistry and conformation change in Rose Bengal molecules. Field-induced

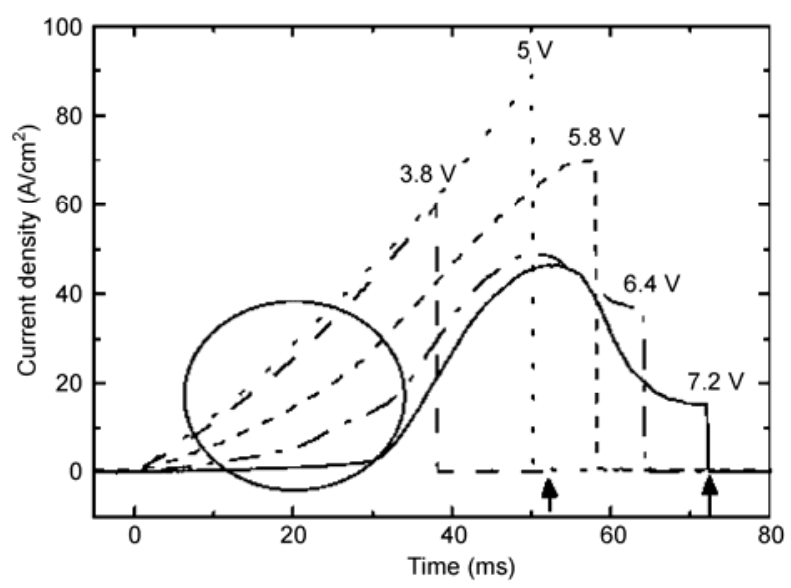

Figure 9 Current difference in the NDR voltage regime. The circled area indicates the variation in current in the low-voltage regime. The arrows indicate the $V_{\max }(5.2 \mathrm{~V})$ and $V_{\min }(7.2 \mathrm{~V})$ [76]. conformation change has also been adopted by various researchers to explain the multilevel effect [85], while still other researchers attributed the multilevel effect of their devices to a filamentary mechanism $[77,83]$. In 2006, Chen et al. [86] reported single layer devices possessing bipolar switching with a negative write voltage and a positive erase voltage. Since the ON state current is dependent on the negative writing voltages, the devices can operate at the multilevel mode by writing different negative voltages as shown in Figure 11. The multilevel mechanism is explained in the same way as the SV mode.

\section{Organic thin-film transistor (OTFT) memory devices}

OTFT-type nonvolatile memories are especially attractive because of their nondestructive readout, complementary integrated circuit architectural compatibility, and single transistor features [87]. Since the report of the first organic field-effect transistor in 1986 [88], there has been tremendous progress in performance and fabrication techniques.

\subsection{Structure and fabrication of OTFT memory devices}

There are generally two basic structures for OTFT memory devices: bottom-gate electrode structure and top-gate electrode structure. Because of the fact that the preparation process of OTFT devices is limited by organic semiconductor materials, especially for organic small molecule materials, it is difficult to fabricate a top-gate electrode OTFT without damaging the active layer and with no degradation of the device. For these reasons, researchers often adopt the bottom-gate electrode structure. According to the relative location between the organic semiconductor and the source/ drain electrodes, the bottom-gate electrode OTFT can be divided into top contact (TC) and bottom contact (BC) structures (Figure 12). Many studies have demonstrated that TC structures have less contact resistance and higher performance than the $\mathrm{BC}$ ones because of the advantages of a larger contact area between the organic layer and the source/drain electrodes, a uniform film with less influence from the image charges, and an additional work function difference.

Currently, two methods are mainly used to fabricate OTFT memory devices, one of which uses ferroelectric materials, such as poly(vinylidene fluoride/trifluoroethylene) (P(VDF/TrFE) ) [89-92] and nylon poly(m-xylylene adipamide) (MXD6) [93], or electret, such as polyvinyl alcohol (PVA) [94] and poly ( $\alpha$-methyl styrene) (P $\alpha \mathrm{MS})$ [95], which is chargeable as gate dielectric material. The memory function is implemented by the polarization of the ferroelectric material or the trapped charges in the electret, which modulate the channel conductance of transistors. The other 


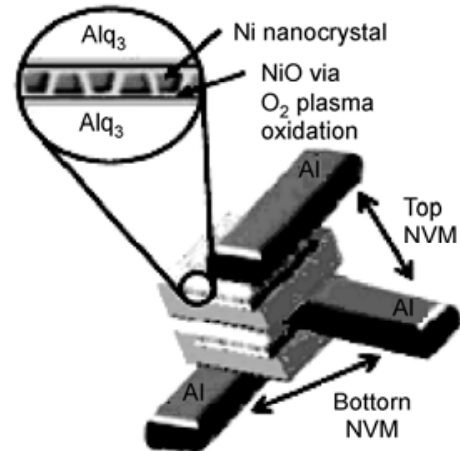

(a)

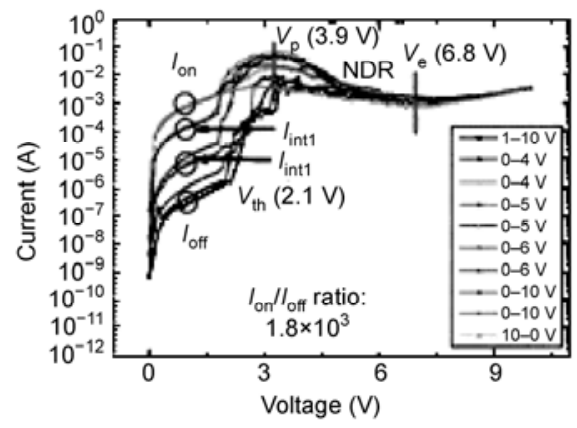

(b)

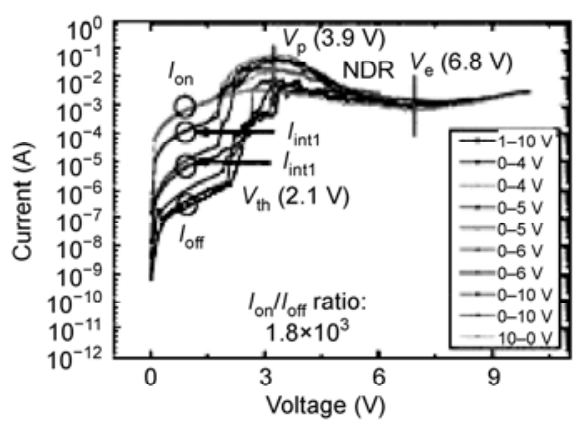

(c)

Figure 10 (a) Perspective view of the vertically double stacked small-molecule nonvolatile $4 \mathrm{~F}^{2}$ memory cell; and dc $I-V$ curves of the (b) lower and (c) upper memory cells [78].

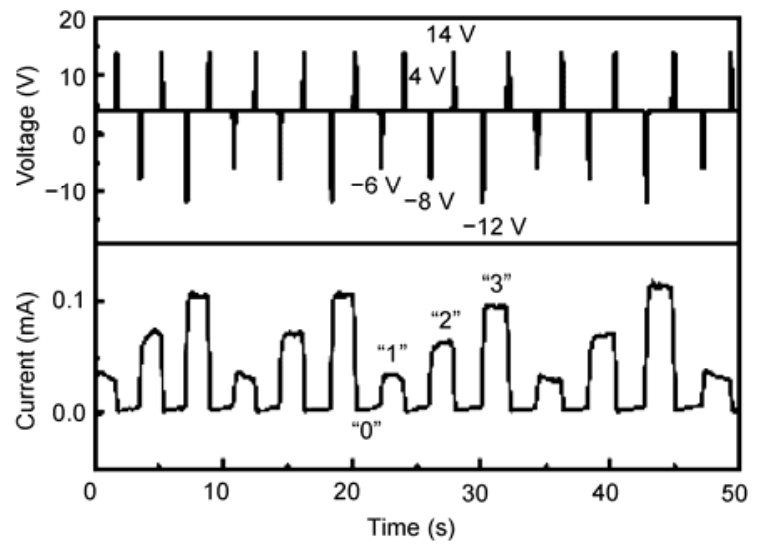

Figure 11 Memory performance of ITO/DAFA(100 nm)/Ag with three different writing voltages: $-6,-18$, and $-12 \mathrm{~V}$, respectively; and erasing and reading voltages of 14 and $4 \mathrm{~V}$, respectively [86].

method intentionally introduces charge traps, e.g., nanocrystals, into the gate dielectrics [96,97].

(1) Ferroelectric and electret OTFT memory. Most of the organic memory transistors reported to date exploit the electric field induced remnant polarization in ferroelectric polymer films. The operation voltage in a ferroelectric memory is generally high because of the utilization of a thick ferroelectric layer to prevent serious gate leakage in an OTFT. Many researchers have tried to reduce the operation voltage through process optimization or utilization of higher dielectric constant materials. In 2004, Schroeder et al. [93] presented OTFT memories with gate insulators based on amorphous, ferroelectric like material poly $(m$-xylylene adipamide) (MXD6) and the devices had an on/off ratio of 200 at a $2.5 \mathrm{~V}$ gate bias, 30 at a $0 \mathrm{~V}$ gate bias, and a retention time of 3 h. In 2005, Naber et al. [90] proposed a ferroelectric OTFT with poly(vinylidene fluoride/trifluoroethylene) $\mathrm{P}(\mathrm{VDF} / \mathrm{TrFE})$ as the ferroelectric polymer and regioregular poly(3-hexylthiophene)(rr-P3HT) as the semiconducting polymer. The programming voltage was reduced to $15 \mathrm{~V}$ by optimizing the ferroelectric layer deposition technique using cyclohexanone as a solvent, which results in thin, smooth and defect-free ferroelectric films. In 2007, Yildirim et al. [92] obtained a high-k gate insulator by properly blending the $\mathrm{P}(\mathrm{VDF} / \mathrm{TrFE})$ polymer solution with barium titanate nanopowder, and reported that the OTFT based on this composite gate dielectric showed low voltage consumption and good memory retention properties]. In recent years, tremendous progress has been achieved in ferroelectric OTFT memories, although there is still much to do in the practical application of nonvolatile ferroelectric OTFT memories.

Hysteresis in the operation of OTFTs has also been observed in cycling OTFTs with electret polymer gate dielectrics. In 2004, Singh et al. [94] reported an OTFT based memory element with an electret as initial gate insulator. They used polyvinyl alcohol (PVA) as the gate insulator and fullerenes as a molecular semiconductor, and observed an on/off drain-source current ratio of $10^{4}$ upon applying a gate voltage, a large shift in $V_{\mathrm{t}}$ by $14 \mathrm{~V}$, and retention times of $15 \mathrm{~h}$. They proposed that the observation of a switchable channel current originated from charge storage in the organic electret. In 2005, Stadlober et al. [91] demonstrated a

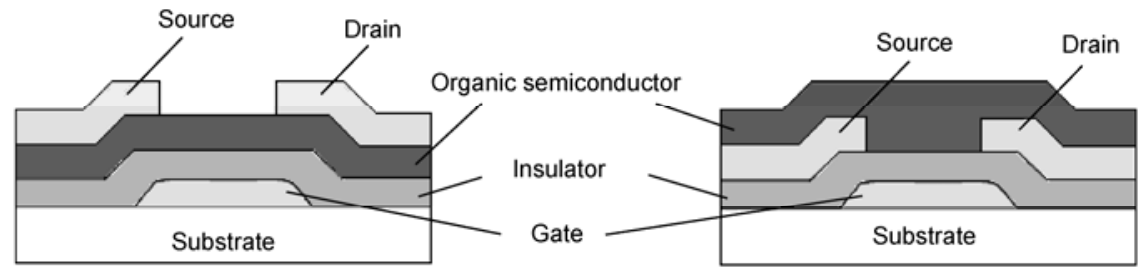

(a)

(b)

Figure 12 Schematics of (a) top contact and (b) bottom contact structures for bottom-gate electrode OTFT. 
high-performance OTFT of TC structure with pentacene as the active material and a double layer polymer electret consisting of $\mathrm{P}(\mathrm{VDF} / \mathrm{TFE} / \mathrm{HFP})$ and PVCi as gate dielectric. $\mathrm{P}(\mathrm{VDF} / \mathrm{TFE} / \mathrm{HFP})$ is a high dielectric constant terpolymer of VDF (vinylidene fluoride), TFE (tetrafluoroethylene), and HFP (hexafluoropropylene). The transistors showed an intrinsic field-effect mobility in the range of $\mu_{\mathrm{i}}=1 \mathrm{~cm}^{2} / \mathrm{V} \mathrm{s}$ and an on/off current ratio of about $10^{5}$. They argued that the morphology of the high-k fluorinated polymer electret strongly influences the electrical properties. In 2006, Baeg et al. [95] demonstrated an OTFT memory with a $\mathrm{SiO}_{2}$ gate insulator that was modified with a thin layer of poly(a-methylstyrene) (PaMS), which acts as a polymeric gate dielectric. This OTFT memory device showed a large memory window (about $90 \mathrm{~V}$ ), a high on/off ratio of $10^{5}$, and a long retention time (more than $100 \mathrm{~h}$ ).

(2) Floating gate memory devices. A floating-gate transistor is a field-effect transistor with two gate electrodes. In addition to the control gate, similar to that in a regular transistor, it has a floating gate embedded in the gate dielectric. When the dielectric is thin enough, electronic charge can be brought onto the floating gate by quantum tunneling or thermal emission when a large enough program voltage is applied between the control gate and the source contact. Charging the floating gate changes the transistor's threshold voltage, because the charge on the floating gate partially screens the electric field between the control gate and the semiconductor. Since the floating gate is completely isolated by the dielectric, charge stored on the floating gate remains there without the need for any applied voltage (nonvolatile memory). To erase the memory, a voltage of opposite polarity is applied, discharging the floating gate through the dielectric. In 2006, Liu et al. [96] fabricated OTFT memory devices on a heavily doped n-type silicon $\left(\mathrm{n}^{+}-\mathrm{Si}\right)$ substrate with a thermally grown oxide layer, of which $\mathrm{n}+-\mathrm{Si}$ functioned as the gate electrode and the oxide layer functioned as the gate dielectric. Gold nanoparticles were deposited on the gate oxide by an electrostatic layer-bylayer self-assembly method. A self-assembled multilayer of polyelectrolytes, together with a thin spin-coated poly(4vinyl phenol) layer, covered the gold nanoparticles and separated them from the poly(3-hexyl thiophene) channel. These transistors showed an on/off ratio over 1500 and data retention time of about 200 s. Recently Kim et al. [98] proposed pentacene-based nonvolatile memory devices using controlled gold nanoparticle/polyelectrolyte composite layers and polymethylmethacrylate (PMMA) as the insulating polymers. A maximum memory window of $34 \mathrm{~V}$ was obtained after applying $\pm 80 \mathrm{~V}$ to the gate, and the memory properties could be maintained for more than one year. Both of these works used an organic/inorganic hybrid structure, whereas our group reported an all-organic memory device based on a copper phthalocyanine $(\mathrm{CuPc})$ thin-film (Figure 13 ), with gold nanocrystals embedded in a polyimide gate dielectric and a window of $20 \mathrm{~V}$ was obtained [97]. Discrete

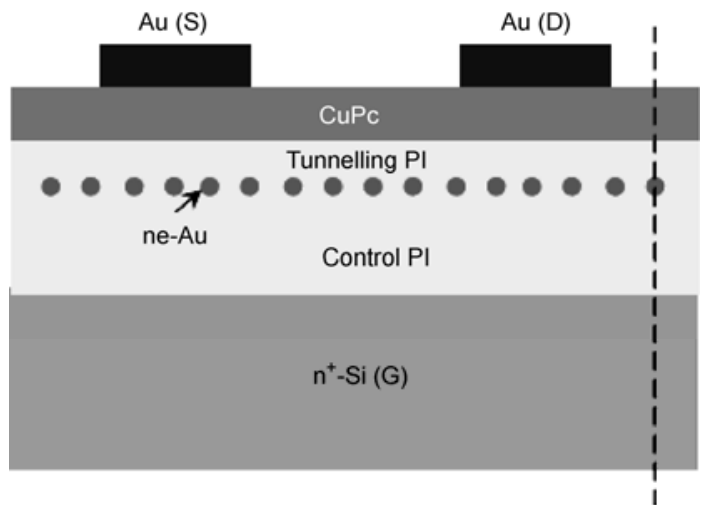

Figure 13 Schematic cross section of the organic nanocrystal memory with three sequential layers of PI/nc-Au/PI (with a thickness of 250/2/50 $\mathrm{nm})$ as a whole insulator layer [97].

gold nanocrystals, which are charged and discharged under proper gate bias, were adopted as the charge storage medium, resulting in the modulation of the channel conductance. To verify the charge storage effect of gold nanocrystals, a control sample without gold nanocrystals was prepared, but negligible hysteresis was found. Current-voltage $(I-V)$ measurements (Figure 14) at room temperature show the memory behavior of the fabricated devices.

\subsection{Applications of OTFT memory devices}

Based on the tremendous progress in fabrication techniques and performance enhancement, the potential of OTFT memories in future applications is attracting more and more research interest, for example, for radio-frequency IDs, smart tags, textile integrated electronics, flexible sensors, and flexible displays. In 2007, Poly IC developed the first OTFT based RFID tags working at $13.56 \mathrm{MHz}$ [99]. Recently, Sekitani et al. [21] developed a sensor matrix by

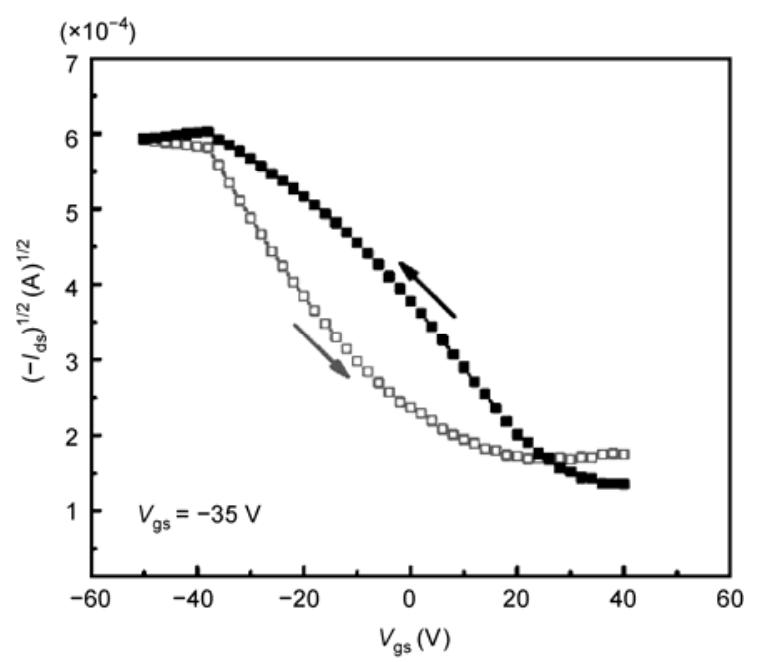

Figure 14 Transfer characteristics with bi-directional scan gate voltage $\left(V_{\mathrm{gs}}\right)$ ranges of a device with nc-Au embedded in polyimide gate dielectrics [97]. 
integrating a flexible array of organic floating-gate transistors with a pressure-sensitive rubber sheet. The flexible floating-gate transistors possessed small program and erase voltages $(-6 \mathrm{~V}$ to $+3 \mathrm{~V})$, and a long retention time of more than $12 \mathrm{~h}$

In the literature there are very few reports on multibit storage of organic thin-film field-effect transistors. In 2009, Guo et al. [100] first demonstrated multibit storage in a single OTFT with pentacene or copper phthalocyaine $(\mathrm{CuPc})$ as the active layer. Because of the use of optical and electrical organic semiconductors, the devices exhibited 2 bits storage in each cell, reversible $V_{\text {th }}$ shifts with electrical and light-assisted programming, and long retention time $(>250$ h). Nevertheless, as the exact mechanism is not precisely understood, further investigation is needed to optimize the performance of these devices.

Tremendous progress has been made in OTFT memories since they were first reported. Currently, OTFT memories with operation voltages of about $2 \mathrm{~V}$, program/erase cycles greater than $10^{3}$, and retention times of a few hundred hours have been achieved. In addition, multibit storage properties as well as integration with other electronic elements of the OTFT memories have also been reported. However, there are still many obstacles, such as operation voltages being generally high, retention time not being long enough for commercial applications, and the mechanism still not being clear. Much experimental and research work still needs to be done.

\section{Summary and outlook}

To understand clearly the differences between two-terminal and OTFT memory, we have summarized the characteristics of each type in Table 1. As the migration rate of organic materials is much lower than traditional inorganic semiconductor materials, this results in high operating voltage and low switching speed of the OTFT, and these features also limit the application of OTFT devices in current highspeed logic circuits.

In summary, investigations of organic memories are growing rapidly, since it is believed that organic memories may provide alternative or supplemental technology to conventional memory technologies facing the problem of miniaturizing from micro to nanoscale. Nevertheless, there are many obstacles limiting the long-term development thereof. The maximal retention time reported to date is several months [60], which is far from the requirements of commercial applications (at least 10 years). Other important device parameters such as on/off ratio, switching time, and cycling endurance are either omitted completely or show large discrepancies in their published values. Switching mechanisms are always speculative, rather than indubitable, and the experimental data are not always reproducible even within the same lab.
Table 1 Comparison of the characteristics of reported two-terminal and OTFT memory devices

\begin{tabular}{lcc}
\hline & $\begin{array}{c}\text { Two-terminal } \\
\text { memory }\end{array}$ & $\begin{array}{c}\text { OTFT } \\
\text { memory }\end{array}$ \\
\hline$I_{\text {on }} / I_{\text {off }}$ ratio (maximum) & $10^{9}[26]$ & $>10^{5}[91]$ \\
Operation voltage (minimum) & $<1 \mathrm{~V}$ & $<2 \mathrm{~V}$ \\
Retention time (maximum) & $>5$ months [60] & $>250 \mathrm{~h} \mathrm{[100]}$ \\
Program, erase cycles (maximum) & $>10^{6}[101]$ & $>10^{3}[96]$ \\
Multilevel storage (bits stored per unit) & $3[78]$ & $2[100]$ \\
\hline
\end{tabular}

The immediate challenges facing organic memories are the fabrication of organic thin film devices with reproducible switching properties and validation of the operational and failure mechanisms. Much effort should be expended to promote the development and possible commercialization of organic memory devices.

This work was supported by the National Basic Research Program of China (2011CB808404, 2009CB939703) and the National Natural Science Foundation of China (60825403, 90607022, and 61001043).

1 Velu G, Legrand C, Tharaud O, et al. Low driving voltages and memory effect in organic thin-film transistors with a ferroelectric gate insulator. Appl Phys Lett, 2001, 79: 659-661

2 Unni K N N, De Bettignies R, Dabos-Seignon S, et al. A nonvolatile memory element based on an organic field-effect transistor. Appl Phys Lett, 2004, 85: 1823-1825

3 Naber R C G, De Boer B, Blom P W M. Low-voltage polymer fieldeffect transistors for nonvolatile memories. Appl Phys Lett, 2005, 87: 203509

4 Stadlober B, Zirkl M, Beutl M, et al. High-mobility pentacene organic field-effect transistors with a high-dielectric-constant fluorinated polymer filmgate dielectric. Appl Phys Lett, 2005, 86: 242902

5 Yildirim F A, Ucurum C, Schliewe R R, et al. Spin-cast composite gate insulation for low driving voltages and memory effect in organic field-effect transistors. Appl Phys Lett, 2007, 90: 083501

6 Singh T B, Marjanovic N, Matt G J, et al. Nonvolatile organic fieldeffect transistor memory element with a polymeric gate electret. Appl Phys Lett, 2004, 85: 5409-5411

7 Liu Z, Xue F, Su Y, et al. Memory effect of a polymer thin-film transistor with self-assembled gold nanoparticles in the gate dielectric. IEEE Trans Nanotechnol, 2006, 5: 379-384

8 Zhen L, Guan W, Shang L, et al. Organic thin-film transistor memory with gold nanocrystals embedded in polyimide gate dielectric. J Phys D: Appl Phys, 2008, 41: 135111

9 Chang C C, Pei Z, Chan Y J. Artificial electrical dipole in polymer multilayers for nonvolatile thin film transistor memory. Appl Phys Lett, 2008, 93: 143302

10 Burrows P E, Forrest S R, Sibley S P. Color-tunable organic lightemitting devices. Appl Phys Lett, 1996, 69: 2959-2961

11 Friend R H, Gymer R W, Holmes A B, et al. Electroluminescence in conjugated polymers. Nature, 1999, 397: 121-128

12 Meyer J, Hamwi S, Bulow T, et al. Highly efficient simplified organic light emitting diodes. Appl Phys Lett, 2007, 91: 113506

13 Brabec C J, Sariciftci N S, Hummelen J C. Plastic solar cells. Adv Funct Mater, 2001, 11: 15-26

14 Peumans P, Uchida S, Forrest S R. Efficient bulk heterojunction photovoltaic cells using small-molecular-weight organic thin films. Nature, 2003, 425: 158-162

15 Gunes S, Neugebauer H, Sariciftci N S. Conjugated polymer-based 
organic solar cells. Chem Rev, 2007, 107: 1324-1338

16 Chen Y, Jung G Y, Ohlberg D A A, et al. Nanoscale molecular-switch crossbar circuits. Nanotechnology, 2003, 14: 462-468

17 Chen Y, Ohlberg D A A, Li X M, et al. Nanoscale molecular-switch devices fabricated by imprint lithography. Appl Phys Lett, 2003, 82: 1610-1612

18 Jung G Y, Ganapathiappan S, Ohlberg D A A, et al. Fabrication of a $34 \times 34$ crossbar structure at $50 \mathrm{~nm}$ half-pitch by UV-based nanoimprint lithography. Nano Lett, 2004, 4: 1225-1229

19 Wu W, Jung G Y, Olynick D L, et al. One-kilobit crossbar molecular memory circuits at $30-\mathrm{nm}$ half-pitch fabricated by nanoimprint lithography. Appl Phys A, 2005, 80: 1173-1178

20 Green J E, Choi J W, Boukai A, et al. A 160-kilobit molecular electronic memory patterned at $10^{11}$ bits per square centimetre. Nature, 2007, 445: 414-417

21 Sekitani T, Yokota T, Zschieschang U, et al. Organic nonvolatile memory transistors for flexible sensor arrays. Science, 2009, 326: 1516-1519

$22 \mathrm{Xu} \mathrm{W}$, Chen G R, Li R J, et al. Two new all-organic complexes with electrical bistable states. Appl Phys Lett, 1995, 67: 2241-2242

23 Chu C W, Ouyang J Y, Tseng J H, et al. Organic donor-acceptor system exhibiting electrical bistability for use in memory devices. Adv Mater, 2005, 17: 1440-1443

24 Tu C H, Lai Y S, Kwong D L. Memory effect in the current-voltage characteristic of 8-hydroquinoline aluminum salt films. IEEE Electron Device Lett, 2006, 27: 354-356

25 Ma L P, Liu J, Yang Y. Organic electrical bistable devices and rewritable memory cells. Appl Phys Lett, 2002, 80: 2997-2999

26 Tondelier D, Lmimouni K, Vuillaume D, et al. Metal/organic/metal bistable memory devices. Appl Phys Lett, 2004, 85: 5763-5765

27 Ouyang J Y, Chu C W, Szmanda C R, et al. Programmable polymer thin film and non-volatile memory device. Nat Mater, 2004, 3: 918-922

28 Prakash A, Ouyang J Y, Lin J L, et al. Polymer memory device based on conjugated polymer and gold nanoparticles. J Appl Phys, 2006, 100: 054309

29 Lee P T, Chang T Y, Chen S Y. Tuning of the electrical characteristics of organic bistable devices by varying the deposition rate of Alq3 thin film. Org Electron, 2008, 9: 916-920

30 Huang J, Virji S, Weiller B H, et al. Nanostructured polyaniline sensors. Chem Eur J, 2004, 10: 1314-1319

31 Majumdar H S, Bandyopadhyay A, Bolognesi A, et al. Memory device applications of a conjugated polymer: Role of space charges. $\mathrm{J}$ Appl Phys, 2002, 91: 2433-2437

32 Bozano L D, Kean B W, Deline V R, et al. Mechanism for bistability in organic memory elements. Appl Phys Lett, 2004, 84: 607-609

33 Ouisse T, Stéphan O. Electrical bistability of polyfluorene devices. Org Electron, 2004, 5: 251-256

34 Oyamada T, Tanaka H, Matsushige K, et al. Switching effect in $\mathrm{Cu}$ :TCNQ charge transfer-complex thin films by vacuum codeposition. Appl Phys Lett, 2003, 83: 1252-1254

35 Mahapatro A K, Agrawal R, Ghosh S. Electric-field-induced conductance transition in 8-hydroxyquinoline aluminum (Alq3). J Appl Phys, 2004, 96: 3583-3585

36 Kuang Y B, Huang R, Tang Y, et al. Novel thermally stable single-component organic-memory cell based on oxotitanium phthalocyanine material. IEEE Electron Device Lett, 2009, 30: 931-933

37 Chen J S, Ma D G. Single-layer organic memory devices based on N, $\mathrm{N}^{\prime}$-di(naphthalene-l-yl)-N,N'-diphenyl-benzidine. Appl Phys Lett, 2005, 87: 023505

38 Liu X H, Ji Z Y, Tu D Y, et al. Organic nonpolar nonvolatile resistive switching in poly(3,4-ethylene-dioxythiophene): Polystyrenesulfonate thin film. Org Electron, 2009, 10: 1191-1194

39 Potember R S, Poehler T O. Electrical switching and memory phenomena in Cu-TCNQ thin. films. Appl Phys Lett, 1979, 34: 405407

40 Gao H, Xue Z, Pang S. Ionized cluster beam deposition of $\mathrm{C}_{60}, \mathrm{Ag}$ TCNQ thin films and electrical switching phenomena. J Phys D: Appl Phys, 1996, 29: 1868-1872
41 Tu D Y, Ji Z Y, Shang L W, et al. Organic, bistable devices with AgTCNQ charge transfer complex by vacuum co-deposition. J Semicondu, 2008, 29: 50-54

42 Ma L P, Xu Q F, Yang Y. Organic nonvolatile memory by controlling the dynamic copper-ion concentration within organic layer. Appl Phys Lett, 2004, 84: 4908-4910

43 Ma L P, Liu J, Yang Y. US patent application. 2001, US 01/17 206

44 Reddy V S, Karak S, Ray S K, et al. Carrier transport mechanism in aluminum nanoparticle embedded AlQ3 structures for organic bistable memory devices. Org Electron, 2009, 10: 138-144

45 Simmons $\mathrm{J}$ G, Verderber R R. New conduction and reversible memory phenomena in thin insulating films. Proc R Soc Lond A, 1967, 301: 77-102

46 Kang S H, Crisp T, Kymissis I, et al. Memory effect from charge trapping in layered organic structures. Appl Phys Lett, 2004, 85: 4666-4668

47 Tang W, Shi H, Xu G, et al. Memory effect and negative differential resistance by electrode-induced two-dimensional single-electron tunneling in molecular and organic electronic devices. Adv Mater, 2005, 17: 2307-2311

48 Ouyang J, Chu C W, Tseng R J, et al. Organic memory device fabricated through solution processing. Proc IEEE, 2005, 93: 1287-1296

49 Prakash A, Ouyang J Y, Lin J L, et al. Polymer memory device based on conjugated polymer and gold nanoparticles. J Appl Phys, 2006, 100: 054309

50 Song Y, Ling Q D, Lim S L, et al. Electrically bistable thin-film device based on PVK and GNPs polymer material. IEEE Electron Device Lett, 2007, 28: 107-110

51 Tseng R J, Baker C O, Shedd B, et al. Charge transfer effect in the polyaniline-gold nanoparticle memory system. Appl Phys Lett, 2007, 90: 053101

52 Carchano H, Lacoste R, Segui Y. Bistable electrical switching in polymer thin films. Appl Phys Lett, 1971, 19: 414-416

53 Henisch H K, Smith W R. Switching in organic polymer films. Appl Phys Lett, 1974, 24: 589-591

54 Segui Y, Ai B, Carchano H. Switching in polystyrene films: Transition from on to off state. J Appl Phys, 1976, 47: 140-143

55 Pender L F, Fleming R J. Memory switching in glow discharge polymerized thin films. J Appl Phys, 1975, 46: 3426-3431

56 Tu C H, Lai Y S, Kwong D L. Memory effect in the current-voltage characteristic of 8-hydroquinoline aluminum salt films. IEEE Electron Device Lett, 2006, 27: 354-356

57 Lai Y S, Tu C H, Kwong D L, et al. Bistable resistance switching of poly(N-vinylcarbazole) films for nonvolatile memory applications. Appl Phys Lett, 2005, 87: 122101

58 Ouyang $\mathrm{M}$, Hou S M, Chen $\mathrm{H} \mathrm{F}$, et al. A new organic-organic complex thin film with reproducible electrical bistability properties. Phys Lett A, 1997, 235: 413-417

59 Gao H J, Sohlberg K, Xue Z Q, et al. Reversible, nanometer-scale conductance transitions in an organic complex. Phys Rev Lett, 2000, 84: $1780-1783$

60 Liu Z C, Xue F L, Su Y, et al. Electrically bistable memory device based on spin-coated molecular complex thin film. IEEE Electron Device Lett, 2006, 27: 151-153

61 Ma Y, Cao X B, Li G, et al. Improving the on/off ratio and reversibility of recording by rational structural arrangement of donor-acceptor molecules. Adv Funct Mater, 2010, 20: 803-810

$62 \mathrm{Ha} \mathrm{H}$, Kim O. Electrode-material-dependent switching characteristics of organic nonvolatile memory devices based on poly(3,4-ethylenedioxythiophene): poly(styrenesulfonate) film. IEEE Electron Device Lett, 2010, 31: 368-370

63 Ouyang J Y, Yang Y. Polymer:metal nanoparticle devices with electrode-sensitive bipolar resistive switchings and their application as nonvolatile memory devices. Appl Phys Lett, 2010, 96: 063506

64 Hua Z Y, Chen G R. A new material for optical, electrical and electronic thin film memories. Vacuum, 1992, 43: 1019-1023

65 Kinoshita K, Tsunoda K, Sato Y H, et al. Reduction in the reset current in a resistive random access memory consisting of $\mathrm{NiO}_{x}$ brought about by reducing a parasitic capacitance. Appl Phys Lett, 2008, 93: 
033506

66 Lee M J, Park Y, Suh D S, et al. Two series oxide resistors applicable to high speed and high density nonvolatile memory. Adv Mater, 2007, 19: 3919-3923

67 Kang B S, Ahn S E, Lee M J, et al. High-current-density $\mathrm{CuO}_{x}$ / $\mathrm{InZnO}_{x}$ thin-film diodes for cross-point memory applications. Adv Mater, 2008, 20: 3066-3069

68 Tu D Y, Liu M, Shang L W, et al. Asymmetric electrical bistable behavior of an eicosanoic acid/zirconium oxide bilayer system with rectifying effect. Appl Phys Lett, 2008, 92: 123302

69 Kim T W, Choi H, Oh S H, et al. Resistive switching characteristics of polymer non-volatile memory devices in a scalable via-hole structure. Nanotechnology, 2009, 21: 025201

70 Sato Y, Tsunoda K, Kinoshita K, et al. Sub-100- $\mu$ A reset current of nickel oxide resistive memory through control of filamentary conductance by current limit of MOSFET. IEEE Trans Electron Device, 2008, 55: 1185-1191

71 Lin H T, Pei Z W, Chen J R, et al. A UV-erasable stacked diodeswitch organic nonvolatile bistable memory on plastic substrates. IEEE Electron Device Lett, 2009, 30: 18-20

72 Teo E Y H, Zhang C F, Lim S L, et al. An organic-based diode-memory device with rectifying property for crossbar memory array applications. IEEE Electron Device Lett, 2009, 30: 487-489

73 Cho B, Kim T W, Song S, et al. Rewritable switching of one diodeone resistor nonvolatile organic memory devices. Adv Mater, 2010, 22: 1228-1232

74 Lai S. Flash memories: Where we were and where we are going. IEEE Inter Electron Devices Meeting Tech Dig, 1998, 971-973

75 Eitan B, Kazerounian R, Roy A, et al. Multilevel flash cells and their trade-offs. IEEE Inter Electron Devices Meeting Tech Dig, 1996, 169-172

76 Majumdar H S, Baral J K, Österbacka R, et al. Fullerene-based bistable devices and associated negative differential resistance effect. Org Electron, 2005, 6: 188-192

77 Lauters M, McCarthy B, Sarid D, et al. Multilevel conductance switching in polymer films. Appl Phys Lett, 2006, 89: 013507

78 Park J G, Nam W S, Seo S H, et al. Multilevel nonvolatile smallmolecule memory cell embedded with Ni nanocrystals surrounded by a NiO tunneling barrier. Nano Lett, 2009, 9: 1713-1719

79 Reddy V S, Karak S, Dhar A. Multilevel conductance switching in organic memory devices based on $\mathrm{AlQ}_{3}$ and $\mathrm{Al} / \mathrm{Al}_{2} \mathrm{O}_{3}$ core-shell nanoparticles. Appl Phys Lett, 2009, 94: 173304

80 Yang G W, Chen H Y, Ma L P, et al. Study of multi-ON states in nonvolatile memory based on metal-insulator-metal structure. Appl Phys Lett, 2009, 95: 203506

81 Rath A K, Pal A J. To induce negative differential resistance in organic devices through a ferroelectric polymer. Org Electron, 2009, 10: 1116-1119

82 Xie X N, Gao X Y, Wang Y Z, et al. Negative differential resistance based on electron injection/extraction in conducting organic films. Appl Phys Lett, 2009, 95: 063301

83 Lauters M, McCarthy B, Sarid D, et al. Nonvolatile multilevel con- ductance and memory effects in organic thin films. Appl Phys Lett, 2005, 87: 231105

84 Bandyopadhyay A, Pal A J. Multilevel conductivity and conductance switching in supramolecular structures of an organic molecule. Appl Phys Lett, 2004, 84: 999-1001

85 Das B C, Pal A J. Switching between different conformers of a molecule: Multilevel memory elements. Org Electron, 2008, 9: 39-44

86 Chen J S, Xu L L, Lin J, et al. Negative differential resistance and multilevel memory effects in organic devices. Semicond Sci Technol, 2006, 21: 1121-1124

87 Auciello O, Scott J F, Ramesh R. The physics of ferroelectric memories. Phys Today, 1998, 51: 22-27

88 Tsumura A, Koezuka H, Ando T. Macromolecular electronic device: Field-effect transistor with a polythiophene thin film. Appl Phys Lett, 1986, 49: 1210-1212

89 Unni K N N, Bettignies R, Dabos-Seignon S, et al. A nonvolatile memory element based on an organic field-effect transistor. Appl Phys Lett, 2004, 85: 1823-1825

90 Naber R C G, Boer B, Blom P W M, et al. Low-voltage polymer field-effect transistors for nonvolatile memories. Appl Phys Lett, 2005, 87: 203509

91 Stadlober B, Zirkl M, Beutl M, et al. High-mobility pentacene organic field-effect transistors with a high-dielectric-constant fluorinated polymer film gate dielectric. Appl Phys Lett, 2005, 86: 242902

92 Yildirim F A, Ucurum C, Schliewe R R, et al. Spin-cast composite gate insulation for low driving voltages and memory effect in organic field-effect transistors. Appl Phys Lett, 2007, 90: 083501

93 Schroeder R, Majewski L A, Grell M. All-organic permanent memory transistor using an amorphous, spin-cast ferroelectric-like gate insulator. Adv Mater, 2004, 16: 633-636

94 Singh T B, Marjanovic N, Matt G J, et al. Nonvolatile organic fieldeffect transistor memory element with a polymeric gate electret. Appl Phys Lett, 2004, 85: 5409-5411

95 Baeg K J, Noh Y Y, Ghim J, et al. Organic non-volatile memory based on pentacene field-effect transistors using a polymeric gate electret. Adv Mater, 2006, 18: 3179-3183

96 Liu Z, Xue F, Su Y, et al. Memory effect of a polymer thin-film transistor with self-assembled gold nanoparticles in the gate dielectric. IEEE Trans Nanotechnol, 2006, 5: 379-384

97 Zhen L, Guan W, Shang L, et al. Organic thin-film transistor memory with gold nanocrystals embedded in polyimide gate dielectric. Appl Phys, 2008, 41: 135111

98 Kim S J, Park Y S, Lyu S H, et al. Nonvolatile nano-floating gate memory devices based on pentacene semiconductors and organic tunneling insulator layers. Appl Phys Lett, 2010, 96: 033302

99 Clemens W. Low cost RFID tags based on printed Electronics. MikroSystemTechnik KONGRESS, 2007

100 Guo Y L, Di C A, Ye S H, et al. Multibit storage of organic thin-film field-effect transistors. Adv Mater, 2009, 21: 1954-1959

101 Ma L P, Pyo S, Ouyang J Y, et al. Nonvolatile electrical bistability of organic/metal-nanocluster/organic system. Appl Phys Lett, 2003, 82: 1419-1421

Open Access This article is distributed under the terms of the Creative Commons Attribution License which permits any use, distribution, and reproduction in any medium, provided the original author(s) and source are credited. 